\title{
Estimación de la longitud utilizando relaciones morfométricas de huesos del cráneo, cintura escapular, otolitos y medidas específicas del cuerpo en Merluccius hubbsi en aguas patagónicas
}

Estimation of body size using morphometric relationships of head bones, pectoral fin bones, otoliths and specific body measurements in Merluccius hubbsi in Patagonian waters

\section{Diego González-Zevallos', Luisa Kuba ${ }^{1}$ y Atila E. Gosztonyi ${ }^{1}$}

\begin{abstract}
${ }^{1}$ Centro Nacional Patagónico CENPAT-CONICET, Blvd. Brown 2915 (U9120ACF), Puerto Madryn, Chubut, Argentina. diegue@cenpat.edu.ar

Abstract.- Merluccius hubbsi is the fish with the largest distributional range along the Argentinian Shelf and one of the most relevant species in the diet of many top predators in the Southwest Atlantic Ocean. Predictive regression equations were calculated to estimate total length using head and pectoral girdle bones lengths and specific body measurements. The 166 specimens studied were obtained in J anuary and February 2008 by coastal hake trawlers operating at Chubut Province, Argentina. Ln- transformed data gave the best fits in the equations obtained. The present study supports the use of head and pectoral girdle bones as an alternative method to identify M. hubbsi and predict its length.
\end{abstract}

Key words: Piscivory, top predators, Argentinian Patagonia

\section{INTRODUCCIÓN}

Merluccius hubbsi (Marini, 1933) es una especie típica de aguas templado-frías, que se distribuye sobre la plataforma continental de Argentina y Uruguay, principalmente entre 80 y $400 \mathrm{~m}$ de profundidad y entre $35^{\circ} \mathrm{S}$ y $54^{\circ} \mathrm{S}$ (Ehrich 1980, Inada 1981, Otero et al. 1982, Bezzi \& Dato 1995, Bezzi et al. 2004). Ocasionalmente, $M$. hubbsi ingresa a aguas brasileñas $\left(23^{\circ} \mathrm{S}\right)$ en respuesta al afloramiento de aguas subantárticas a lo largo de la costa sur de Brasil (Vazzoler \& Iwai 1971, Yesaki 1974¹). Es una especie considerada longeva ya que alcanza edades de 13 y 15 años en los machos y hembras respectivamente (Renzi \& Pérez 1992). Las longitudes estimadas de primera madurez sexual para machos y hembras son de 33,79 y $35,38 \mathrm{~cm}$, correspondientes a edades de 2,61 y 2,67 años respectivamente (Simonazzi 2003). M. hubbsi es una especie desovante parcial que presenta desoves durante casi todo el año, con un período de desove estival más intenso en la zona costera norpatagónica (Pérez Comas 1990, Di Giácomo et al. 1993, Ehrlich \& Ciechomski 1994). M. hubbsi es considerada una especie generalista y oportunista, y presenta canibalismo durante todo el año
(Angelescu \& Prenski 1987, Smith \& Reay 1991, Pitcher \& Alheit 1995, Sánchez \& Rosa 1999). Entre las especies argentinas explotadas, $M$. hubbsi es la que muestra el mayor rango de distribución latitudinal y con la mayor presión pesquera (Cousseau et al. 2004).

Merluccius hubbsi es un importante componente en la dieta de la mayoría de los depredadores tope (Eder \& Lewis 2005), es depredada por condrictios (Angelescu \& Prenski 1987, García de la Rosa \& Sánchez 1998, KoenAlonso et al. 2001, Koen-Alonso et al. 2002), mamíferos marinos (Crespo et al. 1994², Schiavini et al. 1997, Koen Alonso et al. 1998, Koen-Alonso et al. 2000, Crespo et al. 2007), aves marinas (Frere et al. 1996, Scolaro et al. 1999, Punta et al. 2003, González-Zevallos \& Yorio 2006) y posiblemente por peces teleósteos. Dentro de la ecología trófica, es importante determinar lo que un depredador consume, pero también los tamaños corporales de sus presas, asi como sus abundancias, lo que resulta esencial a la hora de definir estrategias de conservación y manejo (Cherel et al. 2000). El presente
${ }^{1}$ Yesaki M. 1974. Os recursos de peixes de arrastro ao largo da costa do Brasil. Programa de Investigación y Desarrollo Pesquero de Brasil. Rio de Janeiro. Documento Técnico 8: 1-45.
${ }^{2}$ Crespo EA, P Nepomnaschy, M Koen \& NA García. 1994. Análisis preliminar de la dieta de mamíferos marinos sobre la base de contenidos estomacales y heces. IV reunión de trabajo en mamíferos acuáticos de América del Sur, 75-88. Valdivia. 
estudio, calculó las ecuaciones de regresión predictivas para estimar la longitud de $M$. hubbsi a partir del tamaño de los otolitos, huesos craneales y de la cintura escapular para la unidad de manejo al sur de $41^{\circ} \mathrm{S}$, incorporando la estimación de longitudes a partir de medidas específicas del cuerpo con información adicional en caso de encontrar especimenes incompletos en los contenidos estomacales.

\section{Material y MÉTOdos}

Se analizaron 166 especimenes de merluza común obtenidos en enero y febrero de 2008 por barcos arrastreros que operaron en la costa de la Provincia de Chubut, Argentina (entre $43^{\circ}$ y $46^{\circ} \mathrm{S}$ ) registrándose su longitud total (media: 33,9 \pm 9,2 cm, rango: 14-62,9 cm). Se extrajeron los huesos craneales y de la cintura escapular de cada espécimen desarticulándolos mediante una combinación de técnicas de disección y agua a $100^{\circ} \mathrm{C}$. La tinción in toto de los huesos se efectuó con solución de rojo de alizarina de acuerdo a la formulación de Hollister (1934). Los huesos se conservaron hasta su medición en glicerina al $100 \%$ previa impregnación y durante los primeros dos días en glicerina al 50\%.

Los huesos seleccionados fueron los que usualmente se encuentran en los contenidos estomacales de los depredadores y permiten diagnosticarlos a nivel de especie. Las mediciones se realizaron con calibre electrónico ( $\pm 0,1 \mathrm{~mm})$ (Figs. 1 y 2), exceptuando la longitud total, medida con ictiómetro $( \pm 1 \mathrm{~mm})$. Para los análisis estadísticos, se verificó el cumplimiento de los supuestos de independencia, normalidad a través de la prueba de Shapiro-Wilks y homocedasticidad y ajuste mediante métodos gráficos basados en los residuales del modelo. La transformación a logaritmo natural (Ln) de las medidas originales, mostró los mejores ajustes para las estimaciones.

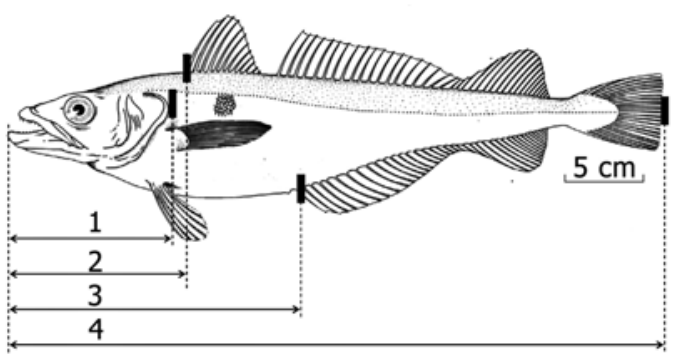

Figura 1. Esquema de las mediciones generales registradas. 1: longitud cabeza; 2: longitud predorsal; 3: longitud preanal y 4: longitud total. (adaptado de Cousseau \& Perrotta 2000) / Schematic view of the general measurements registered. 1: head length; 2: predorsal length; 3: preanal length and 4: total length. (adapted from Cousseau \& Perrotta 2000)
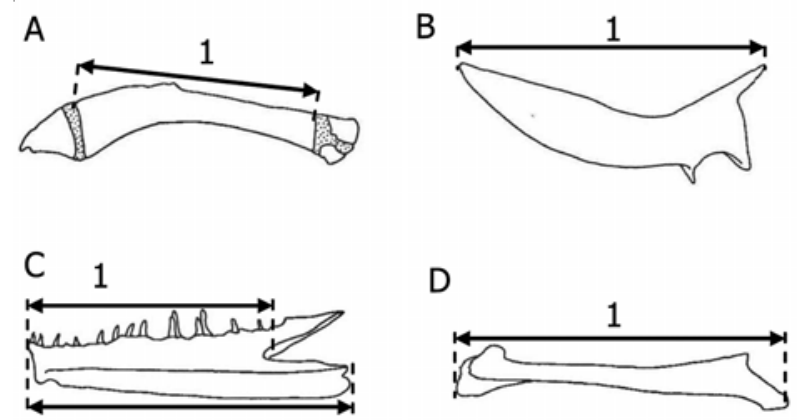

2

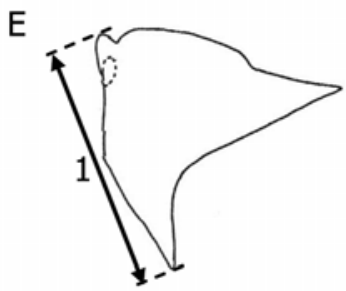

$\mathrm{F}$

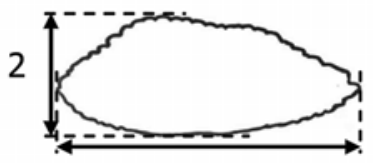

1

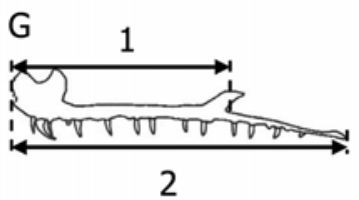

$\mathrm{H}$
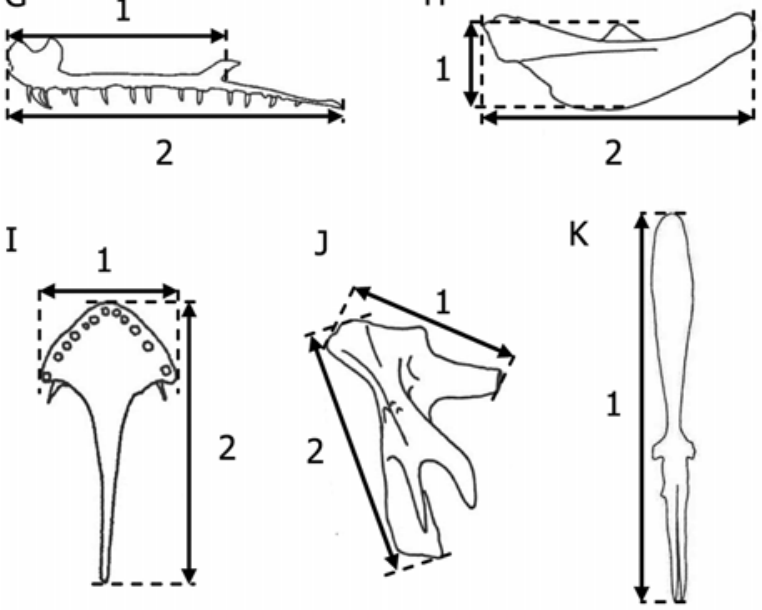

Figura 2. Esquema de las mediciones de los huesos. A: barra hiodea, B: cleitro, C: dentario, D: maxilar, E: opercular, F: otolito, G: premaxilar, H: preopercular, I: vomer, J: hiomandibular y K: parasfenoides (adaptado de Gosztonyi \& Kuba 19963). Los números (1) y (2) representan las mediciones registradas / Schematic view of bone measurements. A: hyoid bar, B: cleithrum, C: dentary, D: maxillary, E: opercular, F: otolith, G: premaxillar, H: preopercular, I: vomer, J: hyomandibular, K: parasphenoid (adapted from Gosztonyi \& Kuba 1996³). The numbers (1) and (2) represent the measurements registered

\footnotetext{
${ }^{3}$ Gosztonyi AE \& L Kuba. 1996. Atlas de huesos craneales y de la cintura escapular de peces costeros patagónicos. Plan de Manejo de la Zona Costera Patagónica. Fundación Patagonia Natural. Informe Técnico 4: 1-29.
} 


\section{RESUltados Y DiscusióN}

Las regresiones mostraron ajustes significativos para todas las variables utilizadas y porcentajes de explicación superiores al $85 \%$. Las ecuaciones de regresión predictivas para los huesos del cráneo, de la cintura escapular, otolitos y medidas específicas del cuerpo con respecto a la longitud, de la merluza se presentan en la Tabla 1.

Tradicionalmente los estudios referidos a la presencia de peces en la dieta de los depredadores se basaban únicamente en el hallazgo y análisis de los otolitos. De esta manera son varios los factores que afectan cualitativa y cuantitativamente los resultados. Los otolitos de diferentes especies podrían ser digeridos diferencialmente en los estómagos de los depredadores, disolviéndose completamente, erosionándose o modificando significativamente su morfología, dificultando las mediciones y/o la identificación taxonómica. Este hecho podría subestimar o ignorar la presencia de ciertas presas e interferir negativamente en las estimaciones de los tamaños originales de las presas (Jobling \& Breiby 1986; Johnstone et al. 1990). Por otro lado, el análisis del material óseo ofrece una mejor y quizás una alternativa más segura para identificar las presas y estimar sus tamaños originales, aún cuando los otolitos hayan sido considerablemente erosionados o disueltos por acción de la digestión (North et al. 1984) o por la acción de los fijadores como la formalina (Gosztonyi \& Kuba 1998).

Tabla 1. Parámetros de las ecuaciones de regresiones predictivas de datos In-transformados vs. la longitud total en $M$. hubbsi $[\ln Y=a+b(\ln X)]$. Los números (1) y (2) se refieren a las mediciones ilustradas en la figura $2, X=$ variable $(\mathrm{mm})$, $\mathbf{Y}=$ longitud total $(\mathrm{cm}), \mathbf{I C}=$ Intervalo de Confianza, $\mathbf{D E}=$ Desviación Estándar, $\mathbf{n}=$ número de ejemplares medidos / Parameters of the predective regression equations of In-transformed data vs. total length in $M$. hubbsi $[(\ln Y=a+b(\ln X)]$. The numbers ( 1 ) and (2) represent the measurements illustrated in figure $2, X=$ variable $(\mathrm{mm}), Y=$ total length $(\mathrm{cm}), I C=$ Confidence Interval, $\mathrm{DE}=$ Standard Deviation, $\mathrm{n}=$ number of specimens measured

\begin{tabular}{|c|c|c|c|c|c|c|}
\hline Variables $(\mathrm{mm})$ & & $\mathrm{n}$ & $r^{2}$ & $\mathrm{a} \pm \mathrm{DE}(\mathrm{IC} 95 \%)$ & $\mathrm{b} \pm \mathrm{DE}(\mathrm{IC} 95 \%)$ & $P$ \\
\hline A barra hiodea & (1) & 142 & 0,94 & $-0,04 \pm 0,08(-0,19 ; 0,11)$ & $1,02 \pm 0,02(0,98 ; 1,06)$ & $<0,001$ \\
\hline B cleitro & (1) & 142 & 0,99 & $-0,36 \pm 0,03(-0,43 ;-0,29)$ & $1,03 \pm 0,01(1,01 ; 1,05)$ & $<0,001$ \\
\hline $\mathrm{C}$ dentario & $\begin{array}{l}\text { (1) } \\
(2)\end{array}$ & $\begin{array}{l}143 \\
143\end{array}$ & $\begin{array}{l}0,97 \\
0,98\end{array}$ & $\begin{array}{c}0,21 \pm 0,05(0,12 ; 0,30) \\
-0,14 \pm 0,04(-0,22 ;-0,05)\end{array}$ & $\begin{array}{l}1,01 \pm 0,01(0,98 ; 1,04) \\
0,99 \pm 0,01(0,96 ; 1,01)\end{array}$ & $\begin{array}{l}<0,001 \\
<0,001\end{array}$ \\
\hline D maxilar & (1) & 143 & 0,99 & $-0,18 \pm 0,04(-0,26 ;-0,11)$ & $1,04 \pm 0,01(1,02 ; 1,06)$ & $<0,001$ \\
\hline E opercular & (1) & 142 & 0,97 & $0,42 \pm 0,04(0,34 ; 0,51)$ & $1,02 \pm 0,01(0,99 ; 1,04)$ & $<0,001$ \\
\hline F otolito & $\begin{array}{l}(1) \\
(2)\end{array}$ & $\begin{array}{l}142 \\
142\end{array}$ & $\begin{array}{l}0,97 \\
0,96\end{array}$ & $\begin{array}{l}0,72 \pm 0,04(0,65 ; 0,80) \\
1,27 \pm 0,04(1,20 ; 1,34)\end{array}$ & $\begin{array}{l}1,04 \pm 0,01(1,01 ; 1,07) \\
1,26 \pm 0,02(1,22 ; 1,30)\end{array}$ & $\begin{array}{l}<0,001 \\
<0,001\end{array}$ \\
\hline G premaxilar & $\begin{array}{l}\text { (1) } \\
\text { (2) }\end{array}$ & $\begin{array}{l}142 \\
143\end{array}$ & $\begin{array}{l}0,98 \\
0,99\end{array}$ & $\begin{array}{c}0,20 \pm 0,04(0,12 ; 0,29) \\
-0,19 \pm 0,04(-0,26 ;-0,12)\end{array}$ & $\begin{array}{l}1,06 \pm 0,01(1,03 ; 1,09) \\
1,05 \pm 0,01(1,03 ; 1,07)\end{array}$ & $\begin{array}{l}<0,001 \\
<0,001\end{array}$ \\
\hline $\mathrm{H}$ preopercular & $\begin{array}{l}\text { (1) } \\
(2)\end{array}$ & $\begin{array}{l}141 \\
140\end{array}$ & $\begin{array}{l}0,95 \\
0,94\end{array}$ & $\begin{array}{l}1,13 \pm 0,05(1,03 ; 1,22) \\
0,27 \pm 0,07(0,13 ; 0,41)\end{array}$ & $\begin{array}{l}0,94 \pm 0,02(0,90 ; 0,98) \\
0,98 \pm 0,02(0,94 ; 1,02)\end{array}$ & $\begin{array}{l}<0,001 \\
<0,001\end{array}$ \\
\hline I vomer & $\begin{array}{l}(1) \\
(2)\end{array}$ & $\begin{array}{l}140 \\
136\end{array}$ & $\begin{array}{l}0,96 \\
0,96\end{array}$ & $\begin{array}{l}0,96 \pm 0,04(0,88 ; 1,05) \\
0,09 \pm 0,06(-0,02 ; 0,21)\end{array}$ & $\begin{array}{l}1,06 \pm 0,02(1,02 ; 1,09) \\
1,03 \pm 0,02(1,0 ; 1,07)\end{array}$ & $\begin{array}{l}<0,001 \\
<0,001\end{array}$ \\
\hline $\mathrm{J}$ hiomandibular & $\begin{array}{l}\text { (1) } \\
(2)\end{array}$ & $\begin{array}{l}143 \\
142\end{array}$ & $\begin{array}{l}0,86 \\
0,94\end{array}$ & $\begin{array}{l}1,39 \pm 0,07(1,25 ; 1,53) \\
0,82 \pm 0,05(0,71 ; 0,93)\end{array}$ & $\begin{array}{l}0,85 \pm 0,03(0,79 ; 0,90) \\
0,95 \pm 0,02(0,91 ; 0,99)\end{array}$ & $\begin{array}{l}<0,001 \\
<0,001\end{array}$ \\
\hline $\mathrm{K}$ parasfenoides & (1) & 139 & 0,99 & $-0,68 \pm 0,03(-0,75 ;-0,61)$ & $1,04 \pm 0,01(1,03 ; 1,06)$ & $<0,001$ \\
\hline longitud cabeza & & 155 & 0,99 & $-1,0 \pm 0,04(-1,08 ;-0,93)$ & $1,03 \pm 0,01(1,01 ; 1,04)$ & $<0,001$ \\
\hline longitud predorsal & & 155 & 0,99 & $-1,14 \pm 0,04(-1,22 ;-1,07)$ & $1,04 \pm 0,01(1,02 ; 1,05)$ & $<0,001$ \\
\hline longitud preanal & & 147 & 0,98 & $-1,18 \pm 0,05(-1,28 ;-1,08)$ & $0,95 \pm 0,01(0,93 ; 0,97)$ & $<0,001$ \\
\hline
\end{tabular}


El presente estudio contribuye a la utilización de huesos del cráneo y la cintura escapular como método alternativo para identificar a nivel de especie y predecir la longitud corporal de $M$. hubbsi. Para obtener información detallada acerca de las relaciones largo-peso y largo-edad de primera madurez sexual en $M$. hubbsi se sugiere complementar el presente estudio con el de Simonazzi (2003).

\section{Agradecimientos}

Al Centro Nacional Patagónico por el apoyo institucional, a la Secretaría de Pesca de la Provincia del Chubut por el apoyo logístico y a Wildlife Conservation Society por el apoyo económico. Al Programa de Observadores a Bordo del Chubut por la obtención de las muestras, en particular a Cristian Marinao y Diego Jara. Un especial agradecimiento a Oscar Biagioni por su colaboración en las mediciones. A los revisores del presente estudio, Néstor García, Lidia Mansur y Pablo Yorio por sus contribuciones.

\section{LITERATURA CITADA}

Angelescu V \& L Prenski. 1987. Ecología trófica de la merluza común del Mar Argentino (Merluccidae, Merluccius hubbsi). Parte 2. Dinámica de la alimentación, analizada sobre la base de las condiciones ambientales, la estructura y las evaluaciones de los efectivos en su área de distribución. Contribución INIDEP 423: 1-248.

Bezzi S \& C Dato. 1995. Conocimiento biológico pesquero del recurso merluza (Merluccius hubbsi) y su pesquería en la República Argentina. Documento Científico INIDEP 4: 1-52. Mar del Plata.

Bezzi S, M Renzi, G Irusta, B Santos, L Tringali, M Ehrlich, F Sánchez, S García de la Rosa, M Simonazzi \& R Castrucci. 2004. Caracterización biológica y pesquera de la merluza (Merluccius hubbsi). En: Sánchez RP \& SI Bezzi (eds). El Mar Argentino y sus recursos pesqueros. Tomo 4. Los peces marinos de interés pesquero. Caracterización biológica y evaluación del estado de explotación, pp. 157205. Publicaciones Especiales INIDEP, Mar del Plata.

Cherel Y, H Weimerskirch \& C Trouvé. 2000. Food and feeding ecology of the neritic-slope forager black-browed albatross and its relationships with commercial fisheries in Kerguelen waters. Marine Ecology Progress Series 207: 183-199.

Crespo EA, MN Lewis \& C Campagna. 2007. Mamíferos marinos: pinnipedios y cetáceos. En: Sánchez RP \& SI Bezzi (eds). El Mar Argentino y sus recursos pesqueros. Tomo 5. El ecosistema marino, pp. 127-150. Publicaciones Especiales INIDEP, Mar del Plata.
Cousseau MB \& RG Perrotta. 2000. Peces marinos de Argentina. Biología, distribución, pesca, 167 pp. Publicaciones Especiales INIDEP, Mar del Plata.

Cousseau MB, AE Gosztonyi, I Elías \& ME Ré. 2004. Estado actual del conocimiento de los peces de la Plataforma Continental Argentina y adyacencias. En: Sánchez RP \& SI Bezzi (eds). El Mar Argentino y sus recursos pesqueros. Tomo 4. Los peces marinos de interés pesquero. Caracterización biológica y evaluación del estado de explotación, pp. 17-38. Publicaciones Especiales INIDEP, Mar del Plata.

Di Giácomo EE, J Calvo, M Perier \& E Morriconi. 1993. Spawning aggregations of Merluccius hubbsi, in Patagonian waters: evidence for a single stock? Fisheries Research 16: 9-16.

Eder EB \& MN Lewis. 2005. Proximate composition and energetic value of demersal and pelagic prey species from the SW Atlantic Ocean. Marine Ecology Progress Series 291: 43-52.

Ehrich S. 1980. Biologische Untersuchungen ueber die Grundfischbestaende vor Argentinien, 59 pp. Institut für Seefischerei, Bundesforschungsanstalt für Fischerei, Hamburg.

Ehrlich M \& JD Ciechomski. 1994. Reseña sobre la distribución de huevos y larvas de merluza (Merluccius hubbsi) basada en veinte años de investigaciones. Frente Marítimo 15(A): 37-50.

Frere E, P Gandini \& V Lichtschein. 1996. Variación latitudinal en la dieta del Pingüino de Magallanes (Spheniscus magellanicus) en la costa Patagónica, Argentina. Ornitología Neotropical 7: 35-41.

García de la Rosa SB \& MF Sánchez. 1998. Alimentación de Squalus acanthias y predación sobre Merluccius hubbsi, en el mar Argentino entre $-34^{\circ} 47^{\prime} \mathrm{S}$ y $-47^{\circ} \mathrm{S}$. Revista de Investigación y Desarrollo Pesquero 11: 119-133.

González-Zevallos D \& P Yorio. 2006. Seabird use of discards and incidental captures at the Argentine hake trawl fishery in the Golfo San Jorge, Argentina. Marine Ecology Progress Series 316: 175-183.

Gosztonyi AE \& L Kuba. 1998. Fishes in the diet of the imperial cormorant Phalacrocorax atriceps in the vicinity of Punta Lobería (Chubut, Argentina). Marine Ornithology 26: 63-68.

Hollister G. 1934. Clearing and dyeing fish for bone study. Zoologica 12(10): 89-101.

Inada T. 1981. Studies on the Merlucciid fishes. Bulletin of the Far Seas Fisheries Research Laboratory 18: 1-72.

Jobling M \& M Breiby. 1986. The use and abuse of fish otoliths in studies of feeding habits of marine piscivores. Sarsia 71: 265-274. 
Johnstone IG, MP Harris, S Wanless \& JA Graves. 1990. The usefulness of pellets for assessing the diet of adult Shags Phalacrocorax aristotelis. Bird Study 37: 5-17.

Koen-Alonso M, EA Crespo, NA García, SN Pedraza \& MA Coscarella. 1998. Diet of dusky dolphins (Lagenorhynchus obscurus), in waters of Patagonia, Argentina. Fishery Bulletin 96(2): 366-374.

Koen-Alonso M, EA Crespo, SN Pedraza, NA García \& MA Coscarella. 2000. Food habits of the south american sea lion, Otaria flavescens, off Patagonia, Argentina. Fishery Bulletin 98(2): 250-263.

Koen-Alonso M, EA Crespo, NA García, SN Pedraza, PA Mariotti, BB Vera \& NJ Mora. 2001. Food habits of Dipturus chilensis (Pisces: Rajidae) off Patagonia, Argentina. ICES Journal of Marine Science 58: 288-297.

Koen-Alonso M, EA Crespo, NA García, SN Pedraza, PA Mariotti \& NJ Mora. 2002. Fishery and ontogenetic driven changes in the diet of the spiny dogfish, Squalus acanthias, in Patagonian waters, Argentina. Environmental Biology of Fishes 63: 193-202.

North AW, MS Burchett, CJ Gilbert \& MG White. 1984. Identification of fish from the southern Ocean by means of otoliths. Bulletin of the British Antarctic Survey 62: 8394.

Otero H, S Bezzi, M Renzi \& G Verazay. 1982. Atlas de los recursos pesqueros demersales del Mar Argentino. Contribución INIDEP 383: 28-42.

Pérez-Comas JA. 1990. Biology and distribution of the Argentine hake (Merluccius hubbsi): considerations on its stock structure, migrations and dynamics of its nursery ground at San Jorge Gulf (Argentina). Ph D Thesis, School of Aquatic and Fishery Sciences, University of Washington, Seattle, 179 pp.
Pitcher TJ \& J Alheit. 1995. What makes a hake? A review of the critical biological features that substaint global hake fisheries. En: Alheit J \& TJ Pitcher (eds). Hake: Fisheries, ecology and markets, pp. 1-14. Chapman \& Hall, London.

Punta G, P Yorio \& G Herrera. 2003. Temporal patterns in the diet and food partitioning in Imperial Cormorants (Phalacrocorax atriceps) and Rock Shag (P. magellanicus) breeding at Bahía Bustamante, Argentina. The Wilson Bulletin 115: 308-316.

Renzi M \& M Pérez. 1992. Un criterio para la determinación de la edad en juveniles de merluza (Merluccius hubbsi) mediante la lectura de otolitos. Frente Marítimo 11(A): 31.

Sánchez MF \& SB García de la Rosa. 1999. Alimentación de Merluccius hubbsi e impacto del canibalismo en la región comprendida entre $-34^{\circ} 50$ 'S y $-47^{\circ} \mathrm{S}$ del Atlántico Sudoccidental. Revista de Investigación y Desarrollo Pesquero 12: 77-93.

Schiavini A, RNP Goodall, AK Lescrauwaet \& M KoenAlonso. 1997. Food habits of Peale's dolphin Lagenorhynchus australis; review and new information. International Whaling Commission Report 47: 827-834.

Scolaro JA, RP Wilson, S Laurenti, MA Kierspel, H Gallelli \& JA Upton. 1999. Feeding preferences of the Magellanic Penguin Spheniscus magellanicus over its breeding range in Argentina. Waterbirds 22: 104-110.

Simonazzi M. 2003. Relación largo-peso y largo-edad de primera madurez sexual de la merluza. Informe Técnico INIDEP 51: 1-26.

Smith C \& P Reay. 1991. Cannibalism in teleost fish. Reviews in Fish Biology and Fisheries 1: 41-64.

Vazzoler G \& M Iwai. 1971. Relatório sôbre prospecção e pesca exploratória na plataforma continental do Río Grande do Sul, 79 pp. Instituto Oceanográfico da Universidad de Sao Paulo, Sao Paulo.

Recibido el 7 de diciembre de 2009 y aceptado el 11 de marzo de 2010 